\title{
Analytical operator solution of master equations describing phase-sensitive processes
}

\author{
A. Vidiella-Barrancoł*, Luis M. Arévalo-Aguilarł, and Hector Moya-Cessa $\oint^{\dagger}$ \\ $\dagger$ †nstituto de Física "Gleb Wataghin", Universidade Estadual de Campinas, 13083-970 Campinas \\ SP Brazil \\ $\ddagger \S I N A O E$, Coordinación de Optica, Apdo. Postal 51 y 216, 72000 Puebla, Pue., Mexico
}

(February 9, 2020)

\begin{abstract}
We present a method of solving master equations which may describe, in their most general form, phase sensitive processes such as decay and amplification. We make use of the superoperator technique.

42.50.Lc, 02.90.+p
\end{abstract}

*E-mail: vidiella@ifi.unicamp.br

†E-mail: hmmc@inaoep.mx 


\section{INTRODUCTION}

Master equations, or evolution equations for reduced density operators are of fundamental importance in the treatment of open systems in quantum theory. They are particularly relevant in the field of quantum optics [1, [2], where generally one has interest in studying the evolution of confined subsystems (a single mode of the electromagnetic field, for instance) "coupled" to external reservoirs. This may model a number of (phase-sensitive or not)

processes, such as the decay of a single mode of the field confined in a lossy cavity [1], as well as amplification processes [2,3]. Interesting applications of phase-sensitive amplification, for example, are the reduction of noise in lasers through the injection of squeezed vacuum [4], as well as noise-free amplification via the two-photon correlated-emission-laser [5].

Nevertheless, complete operator solutions of master equations are not usually presented. Only simple cases such as the decay of a single mode of the field into a vacuum state reservoir $(T=0)$ or stationary regime cases are the ones normally treated [6,2]. Instead, master equations are normally transformed into c-number equations either in the number or coherent state representations, for instance. It would be therefore interesting to obtain a direct solution of the master equation while in its operator form.

\section{THE METHOD}

In this paper we show how to solve exactly master equations of the form

$$
\frac{\partial \hat{\rho}}{\partial t}=\left(\sum_{k=1}^{4} \gamma_{k} \hat{L}_{k}\left(\hat{a}, \hat{a}^{\dagger}\right)\right) \hat{\rho},
$$

where

$$
\begin{aligned}
& \hat{L}_{1}\left(\hat{a}, \hat{a}^{\dagger}\right) \hat{\rho}=2 \hat{a} \hat{\rho} \hat{a}^{\dagger}-\hat{a}^{\dagger} \hat{a} \hat{\rho}-\hat{\rho} \hat{a}^{\dagger} \hat{a} \\
& \hat{L}_{2}\left(\hat{a}, \hat{a}^{\dagger}\right) \hat{\rho}=2 \hat{a}^{\dagger} \hat{\rho} \hat{a}-\hat{a} \hat{a}^{\dagger} \hat{\rho}-\hat{\rho} \hat{a} \hat{a}^{\dagger} \\
& \hat{L}_{3}\left(\hat{a}, \hat{a}^{\dagger}\right) \hat{\rho}=2 \hat{a} \hat{\rho} \hat{a}-\hat{a}^{2} \hat{\rho}-\hat{\rho} \hat{a}^{2},
\end{aligned}
$$

and 


$$
\hat{L}_{4}\left(\hat{a}, \hat{a}^{\dagger}\right) \hat{\rho}=2 \hat{a}^{\dagger} \hat{\rho} \hat{a}^{\dagger}-\hat{a}^{\dagger 2} \hat{\rho}-\hat{\rho} \hat{a}^{\dagger 2}
$$

being $\hat{a}$ and $\hat{a}^{\dagger}$ the bosonic annihilation and creation operators. The $\gamma \mathrm{s}$ are in general complex parameters which may represent gain and decay. For the density operator to be Hermitean, it is necessary that

$$
\gamma_{3}=\left|\gamma_{3}\right| e^{i} \phi=\gamma_{4}^{*}
$$

We may now apply the unitary transformation

$$
\hat{\rho}=\hat{S}(\xi) \hat{\rho}^{\prime} \hat{S}^{\dagger}(\xi)
$$

where $\hat{S}(\xi)$ is the squeeze operator

$$
\hat{S}(\xi)=\exp \left(\frac{\xi^{*} \hat{a}^{2}}{2}-\frac{\xi \hat{a}^{\dagger 2}}{2}\right),
$$

with $\xi=r \exp (i \varphi)$. If we now substitute Eq.(17) into Eq.(11), we obtain an equation for the transformed density operator $\hat{\rho}^{\prime}$, or

$$
\frac{\partial \hat{\rho}^{\prime}}{\partial t}=\left(\sum_{k=1}^{4} \gamma_{k} \hat{L}_{k}\left(\hat{b}, \hat{b}^{\dagger}\right)\right) \hat{\rho}^{\prime},
$$

where

$$
\hat{b}=\hat{S}(\xi)^{\dagger} \hat{a} \hat{S}(\xi)=\mu \hat{a}+\nu \hat{a}^{\dagger},
$$

with

$$
\mu=\cosh (r), \quad \nu=-\sinh (r) \exp (i \varphi) .
$$

We proceed in rewriting Eq.(9) in terms of the original creation and annihilation operators as

$$
\begin{aligned}
\frac{\partial \hat{\rho}^{\prime}}{\partial t}= & \hat{L}_{1}\left(\hat{a}, \hat{a}^{\dagger}\right) \hat{\rho}^{\prime}\left(\gamma_{1} \mu^{2}+\gamma_{2}|\nu|^{2}+\mu \nu\left|\gamma_{3}\right| e^{i \phi}+\mu \nu^{*}\left|\gamma_{3}\right| e^{-i \phi}\right)+ \\
& \hat{L}_{2}\left(\hat{a}, \hat{a}^{\dagger}\right) \hat{\rho}^{\prime}\left(\gamma_{1}|\nu|^{2}+\gamma_{2} \mu^{2}+\mu \nu\left|\gamma_{3}\right| e^{i \phi}+\mu \nu^{*}\left|\gamma_{3}\right| e^{-i \phi}\right)+ \\
& \hat{L}_{3}\left(\hat{a}, \hat{a}^{\dagger}\right) \hat{\rho}^{\prime}\left(\gamma_{1} \mu \nu^{*}+\gamma_{2} \mu \nu^{*}+\mu^{2}\left|\gamma_{3}\right| e^{i \phi}+\left(\nu^{*}\right)^{2}\left|\gamma_{3}\right| e^{-i \phi}\right)+ \\
& \hat{L}_{4}\left(\hat{a}, \hat{a}^{\dagger}\right) \hat{\rho}^{\prime}\left(\gamma_{1} \mu \nu+\gamma_{2} \mu \nu+\nu^{2}\left|\gamma_{3}\right| e^{i \phi}+\mu^{2}\left|\gamma_{3}\right| e^{-i \phi}\right) .
\end{aligned}
$$


We note that one can choose $\phi$ and $r$ in such a way that the last two terms in Eq.(12) become zero. The appropriate choices for these parameters are

$$
\varphi=-\phi
$$

and

$$
\tanh (2 r)=\frac{2\left|\gamma_{3}\right|}{\gamma_{1}+\gamma_{2}}
$$

With the above choice of parameters, then we can write Eq.(12) as

$$
\frac{\partial \hat{\rho}^{\prime}}{\partial t}=\left[\tilde{\gamma}_{1} \hat{L}_{1}\left(\hat{a}, \hat{a}^{\dagger}\right)+\tilde{\gamma}_{2} \hat{L}_{2}\left(\hat{a}, \hat{a}^{\dagger}\right)\right] \hat{\rho}^{\prime}
$$

where

$$
\tilde{\gamma}_{1}=\gamma_{1} \mu^{2}+\gamma_{2}|\nu|^{2}+\mu \nu\left|\gamma_{3}\right| e^{i \phi}+\mu \nu^{*}\left|\gamma_{3}\right| e^{-i \phi}
$$

and

$$
\tilde{\gamma}_{2}=\gamma_{1}|\nu|^{2}+\gamma_{2} \mu^{2}+\mu \nu\left|\gamma_{3}\right| e^{i \phi}+\mu \nu^{*}\left|\gamma_{3}\right| e^{-i \phi}
$$

It follows from the relations above that

$$
\tilde{\gamma}_{1}-\tilde{\gamma}_{2}=\gamma_{1}-\gamma_{2}
$$

and

$$
\tilde{\gamma}_{1}+\tilde{\gamma}_{2}=\left(\gamma_{1}+\gamma_{2}\right) \operatorname{sech}(2 r)
$$

We now write the new master equation (15) in a more convenient form [7]

$$
\frac{\partial \hat{\rho}^{\prime}}{\partial t}=\left(\hat{J}_{1}+\hat{J}_{2}+\hat{J}_{3}-2 \tilde{\gamma}_{2}\right) \hat{\rho}^{\prime}
$$

where we have defined the following super-operators

$$
\hat{J}_{1} \hat{\rho}^{\prime}=2 \tilde{\gamma}_{1} \hat{a} \hat{\rho}^{\prime} \hat{a}^{\dagger}
$$




$$
\hat{J}_{2} \hat{\rho}^{\prime}=2 \tilde{\gamma}_{2} \hat{a}^{\dagger} \hat{\rho}^{\prime} \hat{a}
$$

and

$$
\hat{J}_{3} \hat{\rho}^{\prime}=-\left(\tilde{\gamma}_{1}+\tilde{\gamma}_{2}\right)\left(\hat{a}^{\dagger} \hat{a} \hat{\rho}^{\prime}+\hat{\rho}^{\prime} \hat{a}^{\dagger} \hat{a}\right)
$$

From the equations above, we can write the formal solution of Eq.(20) as

$$
\hat{\rho}^{\prime}(t)=\exp \left(-2 \tilde{\gamma}_{2} t\right) \exp \left[\left(\hat{J}_{1}+\hat{J}_{2}+\hat{J}_{3}\right) t\right] \hat{\rho}^{\prime}(0)
$$

It is not difficult to show that the superoperators $\hat{J}_{1}, \hat{J}_{2}$ and $\hat{J}_{3}$ obey the following commutation relations [8]

$$
\begin{gathered}
{\left[\hat{J}_{2}, \hat{J}_{1}\right] \hat{\rho}^{\prime}=\left[\frac{4 \tilde{\gamma}_{1} \tilde{\gamma}_{2}}{\tilde{\gamma}_{1}+\tilde{\gamma}_{2}} \hat{J}_{3}-4 \tilde{\gamma}_{1} \tilde{\gamma}_{2}\right] \hat{\rho}^{\prime},} \\
{\left[\hat{J}_{1}, \hat{J}_{3}\right] \hat{\rho}^{\prime}=-2\left(\tilde{\gamma}_{1}+\tilde{\gamma}_{2}\right) \hat{J}_{1} \hat{\rho}^{\prime}}
\end{gathered}
$$

and

$$
\left[\hat{J}_{2}, \hat{J}_{3}\right] \hat{\rho}^{\prime}=2\left(\tilde{\gamma}_{1}+\tilde{\gamma}_{2}\right) \hat{J}_{2} \hat{\rho}^{\prime}
$$

In order to disentangle the exponential in Eq.(24), we propose the ansatz

$$
\hat{\rho}^{\prime}(t)=\exp \left(-2 \tilde{\gamma}_{2} t\right) \exp \left[f_{3}(t)\right] \exp \left[f_{2}(t) \hat{J}_{2}\right] \exp \left[f_{0}(t) \hat{J}_{3}\right] \exp \left[f_{1}(t) \hat{J}_{1}(t)\right] \hat{\rho}^{\prime}(0)
$$

By inserting $\hat{\rho}^{\prime}(t)$ in Eq.(28) above into equation in Eq.(20), we obtain the following system of differential equations for the functions $f_{i}$ :

$$
\begin{gathered}
\frac{d f_{0}}{d t}+\frac{4 \tilde{\gamma}_{1} \tilde{\gamma}_{2}}{\tilde{\gamma}_{1}+\tilde{\gamma}_{2}} f_{2}=1 \\
\frac{d f_{1}}{d t} \exp \left[2\left(\tilde{\gamma}_{1}+\tilde{\gamma}_{2}\right)\right]=1 \\
\frac{d f_{2}}{d t}+2 \frac{d f_{0}}{d t} f_{2}+\left(\tilde{\gamma}_{1}+\tilde{\gamma}_{2}\right)+4 \tilde{\gamma}_{1} \tilde{\gamma}_{2} f_{2}^{2}=1
\end{gathered}
$$

and 


$$
\frac{d f_{3}}{d t}-4 \tilde{\gamma}_{1} \tilde{\gamma}_{2} f_{2}=0
$$

Although the system is a non-linear one, its solution is rather straightforward. In order to have the condition $\hat{\rho}^{\prime}(t=0)=\hat{\rho}^{\prime}(0)$ satisfied, we should have $f_{i}(0)=0(i=0,1,2,3)$ as initial conditions for the set of Eqs.(29)-(32). The result is

$$
\begin{gathered}
f_{0}=\frac{\kappa}{\tilde{\gamma}_{1}+\tilde{\gamma}_{2}} t+\frac{1}{\tilde{\gamma}_{1}+\tilde{\gamma}_{2}} \ln \left(\frac{\kappa+\Gamma(t)}{\kappa}\right), \\
f_{1}=f_{2}=\frac{1}{2} \frac{\Gamma(t)}{\kappa+\Gamma(t)},
\end{gathered}
$$

and

$$
f_{3}=2 \tilde{\gamma}_{2} t-\ln \left(\frac{\kappa+\Gamma(t)}{\kappa}\right),
$$

where $\Gamma(t)=(1-\exp [-2 \kappa t]) \tilde{\gamma}_{2}$.

By finally defining the dimensionless superoperators

$$
\hat{L}_{-} \hat{\rho} \doteq \underline{\hat{b}} \hat{\rho} \underline{\hat{b}}^{\dagger}, \quad \hat{L}_{+} \hat{\rho} \doteq \underline{\hat{b}}^{\dagger} \hat{\rho} \underline{\hat{b}}, \quad, \quad \hat{L}_{3} \hat{\rho} \doteq \underline{\hat{b}} \underline{\hat{b}} \hat{\rho}+\hat{\rho} \hat{b}^{\dagger} \underline{\hat{b}}+\underline{\rho}
$$

where the superoperators $\hat{L}_{-}, \hat{L}_{+}$and $\hat{L}_{3}$ obey the commutation relations $\left[\hat{L}_{-}, \hat{L}_{+}\right] \hat{\rho}=\hat{L}_{3} \hat{\rho}$ and $\left[\hat{L}_{3}, \hat{L}_{ \pm}\right] \hat{\rho}= \pm 2 \hat{L}_{ \pm} \hat{\rho}$, we can write the solution of (11) in the form,

$$
\hat{\rho}(t)=e^{\kappa t} e^{\frac{\Gamma(t)}{\kappa+\Gamma(t)} \hat{L}_{+}}\left[\frac{\kappa e^{-\kappa t}}{\kappa+\Gamma(t)}\right]^{\hat{L}_{3}} e^{\frac{\tilde{\gamma}_{1}}{\tilde{\gamma}_{2}} \frac{\Gamma(t)}{\kappa+\Gamma(t)} \hat{L}_{-}} \hat{\rho}(0),
$$

where in (36) we have defined

$$
\underline{\hat{b}}=\hat{S}(\xi) \hat{a} \hat{S}^{\dagger}(\xi)=\mu \hat{a}-\nu \hat{a}^{\dagger}
$$

which should be compared with Eq. (10). Note that if in Eq. (37) we set the parameters $\xi=0$ and $\tilde{\gamma}_{2}=0$ we recover the usual solution for a dissipative cavity at zero temperature as well as for the phase insensitive case.

We have therefore obtained the full solution of the master equation for $\hat{\rho}^{\prime}$ [see Eq.(20)], given by (37). We remind that we still have to apply the transformation in Eq.(17) in order to recover the original density operator $\hat{\rho}$. 
It would be appropriate now to show which interesting cases could be easily treated by employing our solution. For instance, if we make $\gamma_{1}=\gamma(\bar{n}+1) / 2, \gamma_{2}=\gamma \bar{n} / 2, \gamma_{3}=$ $-\gamma M^{*} / 2$ and $\gamma_{4}=-\gamma M / 2$, we will obtain the standard equation describing the decay of a bosonic field into a phase-sensitive reservoir [2]. If on the other hand $\gamma_{1}=\mathcal{A} \bar{n} / 2$ and $\gamma_{2}=\mathcal{A}(\bar{n}+1) / 2$, but keeping $\gamma_{3}=-\mathcal{A} M^{*} / 2$ and $\gamma_{4}=-\mathcal{A} M / 2$, we will have the standard equation describing phase-sensitive amplification [2]. The constants $\gamma$ and $\mathcal{A}$ represent decay and gain, respectively, and $M(\bar{n})$ are connected with phase-sensitive (or not) reservoir fluctuations. Each of this two cases are relevant in the problems of field decay and in the reduction of noise in lasers 沺. We remark that our operator solution turns unnecessary the convertion of the master equation into sometimes cumbersome c-number equations.

Let us finally consider as an example an initial density matrix of the form

$$
\hat{\rho}(0)=\hat{S}(\xi)|0\rangle\langle 0| \hat{S}^{\dagger}(\xi),
$$

or a squeezed vacuum state. After inserting it into (37) it is easy to show that the density operator of the field at a time $t$ may be written as

$$
\hat{\rho}(t)=\frac{\kappa}{\kappa+\Gamma(t)} \sum_{m=0}^{\infty}\left[\frac{\Gamma(t)}{\kappa+\Gamma(t)}\right]^{m}|m, \xi\rangle\langle m, \xi|,
$$

where $|m, \xi\rangle=\hat{S}(\xi)|m\rangle$ are the squeezed number states [9,10]. We may now to follow the state's evolution in phase space, using for instance the $Q$-function [12], defined as

$$
Q(t)=\frac{1}{\pi}\langle\beta|\hat{\rho}(t)| \beta\rangle,
$$

where $|\beta\rangle$ is a coherent state. In our case the $Q$-function is given by

$$
Q(t)=\frac{1}{\pi} \frac{\kappa}{\kappa+\Gamma(t)} \sum_{m=0}^{\infty}\left[\frac{\Gamma(t)}{\kappa+\Gamma(t)}\right]^{m}|\langle\beta \mid m, \xi\rangle|^{2},
$$

with

$$
\langle\beta \mid m, \xi\rangle=\exp \left(-|\beta|^{2} / 2\right) \sum_{n=0}^{\infty} G_{n m}\left(-\beta^{*}\right)^{n},
$$

and where [9] 


$$
G_{n m}= \begin{cases}(-1)^{n+m / 2}\left(\frac{m !}{\cosh r}\right)^{1 / 2}\left(\frac{\tanh r}{2}\right)^{n+m / 2} \sum_{l=0} \frac{\left(\frac{-4}{\sinh ^{2} r}\right)^{l}}{(2 l) !\left(\frac{n}{2}-l\right) !\left(\frac{m}{2}-l\right) !} & \text { if } n, m \text { even } \\ (-1)^{n+m / 2-3 / 2}\left(\frac{m !}{\cosh ^{3} r}\right)^{1 / 2}\left(\frac{\tanh r}{2}\right)^{(n+m) / 2-1} \sum_{l=0} \frac{\left(\frac{-4}{\sinh ^{2} r}\right)^{l}}{(2 l+1) !\left(\frac{n-1}{2}-l\right) !\left(\frac{m-1}{2}-l\right) !} & \text { if } n, m \text { odd } \\ 0 & \text { otherwise }\end{cases}
$$

In figure 1 it is illustrated the $Q$-function in (42) for different times. At $t=0$ we have the $Q$ function of the initial squeezed vacuum state. As time goes on, thermal fluctuations of the reservoir cause a "spread" of the $Q$ function, associated to the increase in the quadrature noise. Nevertheless, due to the phase-sensitive properties of the reservoir, the noise assymmetry characteristic of squeezed states is somehow preserved, as it may be seen in figure 1.

\section{CONCLUSIONS}

To summarize, we have presented an alternative way of treating problems involving certain types of master equations. Our operator solution may be useful to retrieve any sort of information related to the evolution of sub-systems having specific initial conditions. This contrasts with the usual approaches, where only partial information, such as mean values of amplitudes and/or diffusion coefficients is normally obtained.

Note added in proof: While preparing the answer to the referee, we became aware of the paper by Dung and Knöll [11] where they also solve Eq. (1) but using Fokker-Planck equations instead. We would like to stress that our method of solving the master equation (西) is considerably more straightforward and simple.

\section{ACKNOWLEDGMENTS}

The authors would like to thank the Mexican Consejo Nacional de Ciencia y Tecnología (CONACyT) and the Brazilian Conselho Nacional de Desenvolvimento Científico e Tecnológico (CNPq) for support. 


\section{FIGURES}
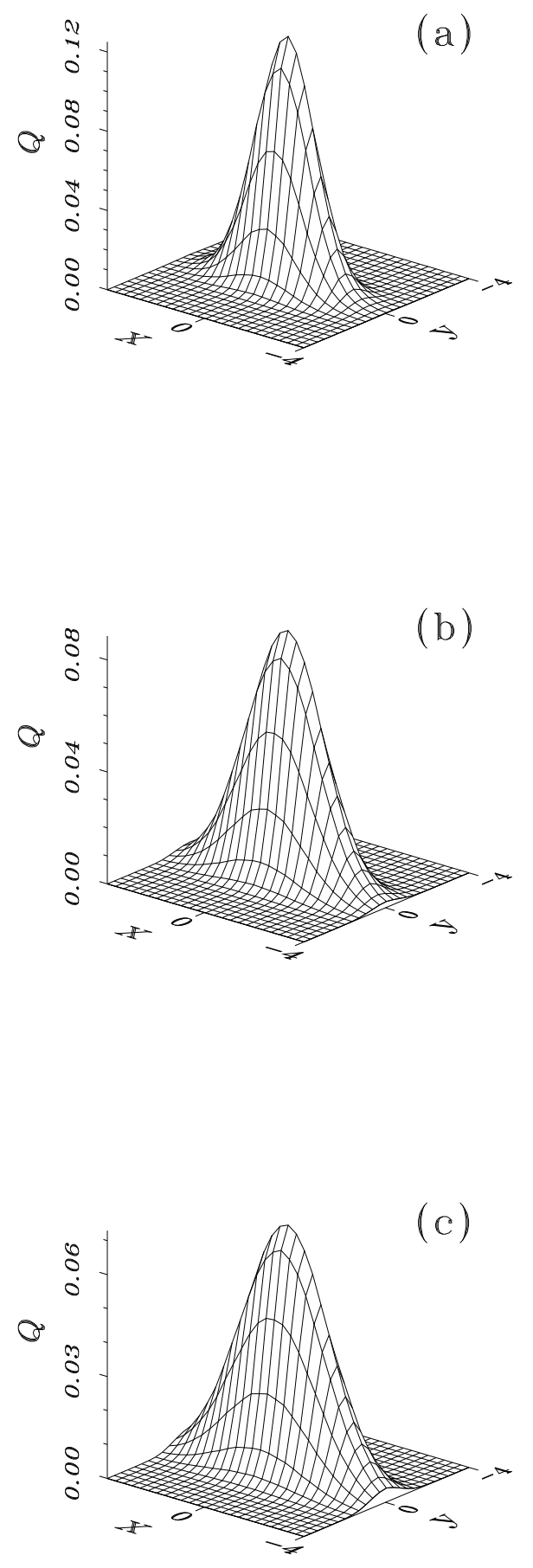

FIG. 1. $Q$ function of the cavity field initially in a squeezed vacuum state. a) at $\kappa t=0$, b) at $\kappa t=0.5$, and c) at $\kappa t=1.0$. It has been taken $\xi=r=0.7$ real, and $\tilde{\gamma}_{2}=1$. 


\section{REFERENCES}

[1] W. H. Louisell, Quantum Statistical Properties of Radiation (Wiley, New York, 1973) p. 331.

[2] M.O. Scully and M.S. Zubairy, Quantum Optics (Cambridge UP, London, 1997) p. 450; G.J. Milburn and D.F. Walls, Quantum Optics (Springer, Berlin, 1994) p. 91.

[3] H. Huang, S.Y. Zhu, and M.S. Zubairy, Phys. Rev. A 53, 1027 (1996).

[4] J. Gea-Banacloche, Phys. Rev. Lett. 59, 543 (1987).

[5] M.O. Scully and M.S. Zubairy, Opt. Commun., 69, 37 (1988).

[6] S.M. Barnett and P.L. Knight, Phys. Rev. A 33, 2444 (1986); see also S.M. Barnett and P.M. Radmore, Methods in Theoretical Quantum Optics (Oxford UP, Oxford, 1997) p. 167.

[7] L.M. Arévalo-Aguilar and H. Moya-Cessa, Quantum and Semiclassical Optics 10, 671 (1998).

[8] Note that the commutators $\left[\hat{J}_{i}, \hat{J}_{j}\right]$ resemble those belonging to the $S U(2)$ group.

[9] M.V. Satyanarayana, Phys. Rev. D 32, 400 (1985).

[10] P. Kral, J. of Mod. Optics 37, 889 (1990); M.S. Kim, F.M.A. de Oliveira and P.L.Knight, Optics Commun. 72, 99 (1989).

[11] H.T. Dung and L. Knöll, J. of Mod. Optics 46, 859 (1999).

[12] M. Hillery, R.F. O'Connell, M.O. Scully, and E.P. Wigner, Phys. Rep. 106, 121 (1984). 